\title{
White Paper: Interventional MRI: Current Status and \\ Potential for Development Considering Economic \\ Perspectives, Part 1: General Application \\ White Paper: Interventionelle MRT: Status Quo und \\ Entwicklungspotenzial unter ökonomischen Perspektiven, \\ Teil 1: generelle Anwendungen
}

Authors (alphabetical order)

Jörg Barkhausen ${ }^{1}$, Thomas Kahn², Gabriele A. Krombach ${ }^{3}$, Christiane K. Kuhl'4, Joachim Lotz ${ }^{5}$, David Maintz ${ }^{6}$, Jens Ricke, Stefan O. Schönberg ${ }^{8}$, Thomas J. Vogl ${ }^{9}$, Frank K. Wacker $^{10}$

German Association of Chairmen in Academic Radiology (KLR)

G. Adam, G. Antoch, J. Barkhausen, M. Beer, T. Bley, A. Bücker, C. Düber, M. Forsting, B. Hamm, K. Hauenstein, W. Heindel,

N. Hosten, O. Jansen, T. Kahn, H. U. Kauczor, G. A. Krombach, C. Kuhl, M. Langer, M. Laniado, J. Lotz, A. Mahnken, D. Maintz,

K. Nikolaou, M. Reiser, J. Ricke, E. Rummeny, H. Schild, S. Schönberg, R. P. Spielmann, C. Stroszczynski, U. Teichgräber, M. Uder,

T. J. Vogl, F. K. Wacker

Affiliations

1 Department of Radiology and Nuclear Medicine, University Hospital Schleswig Holstein Luebeck Campus, Germany

2 Clinic and Policlinic for Diagnostic and Interventional Radiology, Leipzig, Germany

3 Department of Radiology, University Hospital Giessen, Justus Liebig University, Giessen, Germany

4 Department of Diagnostic and Interventional Radiology, University Hospital RWTH Aachen, Germany

5 Institute for Diagnostic and Interventional Radiology, Georg-August-University Goettingen, Germany

6 Department of Radiology, University Hospital of Cologne, Cologne, Germany

7 Department of Radiology and Nuclear Medicine, University Hospital Magdeburg, Germany

8 Institute of Clinical Radiology and Nuclear Medicine, University Medical Center Mannheim, Medical Faculty Mannheim, University of Heidelberg, Mannheim, Germany

9 Department of Diagnostic and Interventional Radiology, University Hospital Frankfurt, Germany

10 Department for Diagnostic and Interventional Radiology, Hannover Medical School, Hannover, Germany

Key words

interventional MRI, MR-guided biopsy, MR-guided ablation, MR-guided endovascular procedures

received 20.10.2016

accepted 12.04.2017
Bibliography

DOI https://doi.org/10.1055/s-0043-110011

Published online: 2017 | Fortschr Röntgenstr 2017; 189:

611-623 @ Georg Thieme Verlag KG, Stuttgart · New York, ISSN 1438-9029

Correspondence

Prof. Gabriele A. Krombach

Departrnent of Radiology

University Hospital Giessen, Justus Liebig University Giessen, Klinikstraße 33, 35392 Giessen, Germany

Tel.: ++49/6 41/98541800

Fax: ++ 49/6 41/98541809

gabriele.krombach@uniklinikum-giessen.de

\section{ABSTRACT}

Background MRI is attractive for the guiding and monitoring of interventional procedures due to its high intrinsic soft tissue contrast and the possibility to measure physiologic parameters like flow and cardiac function.

Method The current status of interventional MRI for the clinical routine was analyzed.

Results The effort needed for the development of MR-safe monitoring systems and instruments initially resulted in the application of interventional MRI only for procedures that could not be performed by other means. Accordingly, biopsy of lesions in the breast, which are not detectable by other modalities, has been performed under MRI guidance for decades. Currently, biopsies of the prostate under MRI guidance are established in a similar fashion. At many sites blind biopsy has already been replaced by MR-guided biopsy or at least by the fusion of MR images with ultrasound. Cardiovascular 
interventions are performed at several centers for ablation as a treatment for atrial fibrillation.

Conclusion Interventional MRI has been established in the clinical routine for a variety of indications. Broader application can be expected in the clinical routine in the future owing to the multiple advantages compared to other techniques.

\section{Key points}

- Due to the significant technical effort, MR-guided interventions are only recommended in the long term for regions in which MRI either facilitates or greatly improves the intervention.

- Breast biopsy of otherwise undetectable target lesions has long been established in the clinical routine. Prostate biopsy is currently being introduced in the clinical routine for similar reasons. Other methods such as MR-guided focused ultrasound for the treatment of uterine fibroids or tumor ablation of metastases represent alternative methods and are offered in many places.

- Endovascular MR-guided interventions offer advantages for a number of indications and have already been clinically established for the treatment of children with congenital heart defects and for atrial ablation at individual centers. Greater application can be expected in the future.

\section{Citation format}

- Barkhausen J, Kahn T, Krombach GA et al. White Paper: Interventional MRI: Current Status and Potential for Development Considering Economic Perspectives, Part 1: General Application. Fortschr Röntgenstr 2017; 189: 611-623

\section{ZUSAMMENFASSUNG}

Hintergrund Die MRT ist aufgrund des hohen intrinsischen Weichteilkontrasts, der Möglichkeit der multiplanaren Aufnahme und der Messung physiologischer Parameter (Fluss, Perfusion, Bewegung etc.) für Steuerung und Überwachung von Interventionen attraktiv.

Methoden Diese Arbeit beschreibt den derzeitigen Stellenwert der interventionellen MRT in der klinischen Routine und die Entwicklungsperspektiven.

Ergebnisse Der hohe Aufwand, Überwachungssysteme und MR-sichere Instrumente zu entwickeln, hat trotz der Vorteile dazu geführt, dass diese Methode sich zunächst nur für Indikationen etablieren konnte, die ihrerseits mittels anderer Modalitäten nicht durchgeführt werden können. So wird die interventionelle MRT seit Dekaden für die Biopsie von MR-suspekten Veränderungen in der Brust eingesetzt. Derzeit wird die MRT in ähnlicher Weise für die Prostata etabliert: die Blindbiopsie ist meistenorts bereits durch die MR-geführte gezielte Probenentnahme oder zumindest durch die Fusion von MRTAufnahmen mit dem Ultraschall ersetzt. Kardiovaskuläre Interventionen werden an einigen Zentren bereits in der Routine zur Ablation bei Vorhofflimmern MR-gesteuert durchgeführt.

Schlussfolgerung Die interventionelle MRT ist für eine Vielzahl von Indikationen bereits in der klinischen Routine etabliert. Aufgrund der Vorteile des Verfahrens im Vergleich zu anderen Techniken ist eine weitere Ausweitung des Einsatzes der interventionellen MRT in Zukunft zu erwarten.

\section{Introduction}

MRI has an intrinsically high soft-tissue contrast and can be used to measure physiological parameters, such as blood flow (flow rate and flow volume), diffusion, temperature, and movement. Since its introduction into the clinical routine in the $1980 \mathrm{~s}$, it has also been used for guiding and monitoring interventions. However, in contrast to $\mathrm{CT}$ and ultrasound, the use of MRI for guiding interventions requires significant technical effort. Due to the disruptive effect of the strong main magnetic field and the alternating electromagnetic fields, the monitoring of patients during an intervention by measuring ECG signals, oxygen saturation, or blood pressure requires special equipment. These monitoring systems must not interfere with the MRI scan and must be MR-safe (according to DIN EN 62570:2010 - 05). The list of requirements regarding monitoring equipment provides an indication of the development effort, with suitable monitoring equipment representing only one of the components necessary for MR-guided interventions.

In addition to MRI safety, all catheters and instruments must be adapted to this modality. To ensure MRI safety, metal must be largely avoided since, depending on the length and orientation to the main magnetic field, it can heat up and consequently even result in burns. Achieving rigidity and torsion stability of catheters or guide wires without the use of metal for reinforcement represents a technical challenge. In the case of needles, breaking resistance is one of the more difficult properties to achieve for MR-safe designs. Moreover, instruments must be able to be effectively visualized without adjacent structures being superimposed by artifacts. The tip must be able to be identified and definitively differentiated from the shaft since MRI as a cross-sectional imaging method typically uses individual slices even for guiding interventions and does not use projections as in fluoroscopy and angiography. As a result, it is necessary to be able to clearly identify whether the instrument tip is located in the slice or the shaft is simply passing through the slice. Flexible instruments must have labeling on the shaft to show whether a distal or proximal shaft segment is visualized in a particular slice. To obtain CE certification for interventional instruments, they must be MR-safe as well as visible under fluoroscopy. This is required to ensure that it is possible to switch to fluoroscopy in the event of a necessary termination of MRI guidance so that catheters or instruments can at least be removed from the patient. As a result of these requirements, the development and production of catheters, guide wires and other instruments are challenging. In addition to image guidance via MRI, interventions can also be guided via other modalities such as ultrasound, fluoroscopy, and CT. In the case of these other modalities, interventionalists can select from a range of available 

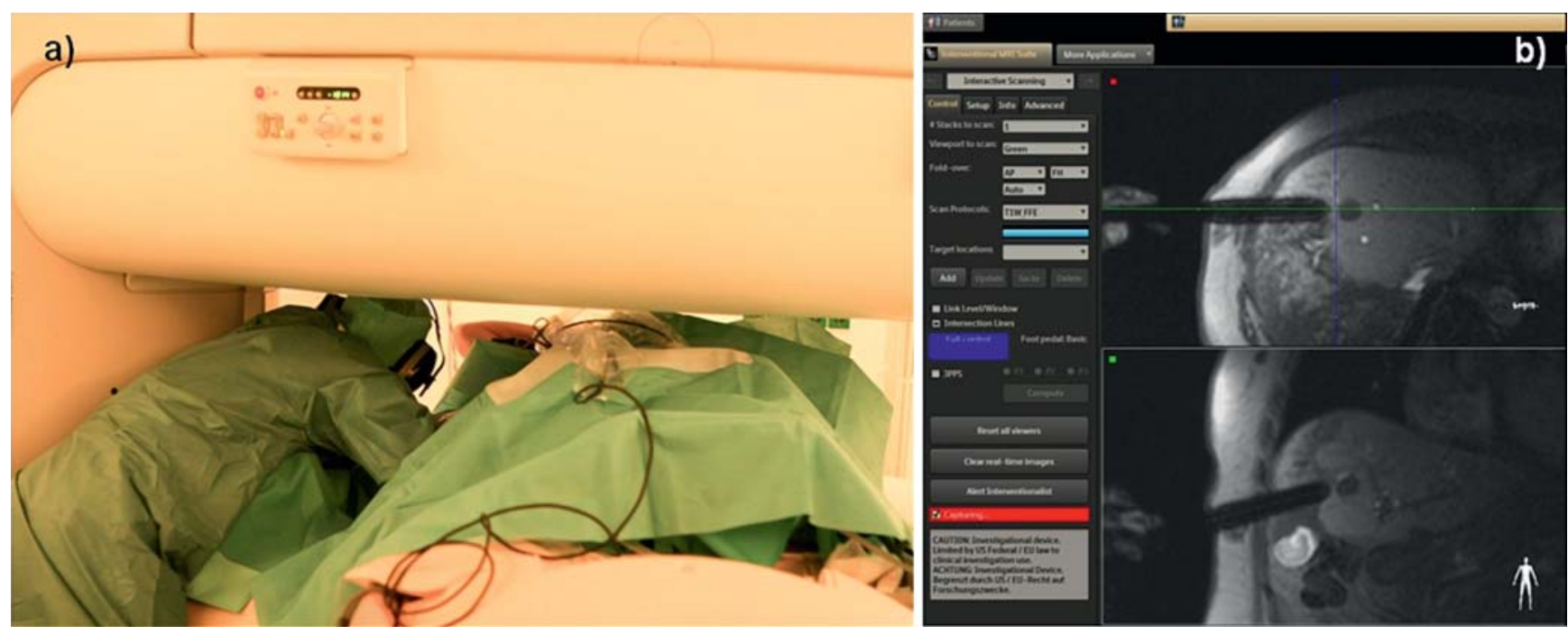

- Fig. 1 MR-guidede radio frequence ablation (RFA) of a liver metastasis. a An open scanner configuration provides sufficient space for the patient and the interventionalist. $\mathbf{b}$ Interactive program for planning and monitoring of the intervention. Exact positioning of the needle is possible by picturing it in two planes.

instruments and the intervention environment is uncomplicated with respect to patient monitoring.

For this reason, the transfer of interventional MRI from applications in animal experiments to the clinical routine was slow to happen. Yet, MRI allows target visualization and intervention guidance that cannot be achieved with any other method. Good examples of this include biopsy of breast lesions that cannot be visualized via ultrasound or mammography and laser ablation of liver tumors with MRI monitoring of the region with a successful increase in temperature. Interventional MRI has long been included in the clinical routine for these indications. Similar advantages for many other procedures that can only be carried out via interventional MRI justify the significant development effort. For the inclusion of the method in the clinical routine, it must be determined whether cost coverage can be ensured. All aspects of DRG reimbursement must be included in such an analysis. This overview describes interventional MRI methods and current fields of indication. Finally, new development prospects are identified and new fields of application are presented.

\section{Interstitial free-hand interventions on open scanners}

An open MRI scanner design improves access to the patient, thus allowing the use of real-time sequences for fluoroscopy techniques. [2, 3]. As a result, dedicated stereotactic and robotic systems are not necessary and the intervention can be conducted using the freehand technique ( $\triangleright$ Fig. 1).

Interactive programs for the quick setting and planning of different planes are available. A display screen on the scanner allows monitoring of the intervention. The intervention sequence is set up so that it visualizes the target lesion as a single slice with optimum contrast and acquires a complete image every second. Performing the intervention in two spatial directions allows safe and easy alignment of the needle since deviations from one plane to the other can be detected and immediately corrected [4].

The diagnostic and therapeutic spectrum is broad and includes a wide range of interstitial percutaneous interventions: The method is currently used for periradicular therapy (PRT), intraabdominal drain placement including nephrostomy, diagnostic biopsy of unclear lesions primarily of the breast and liver and for local ablation of malignant hepatic and renal masses [5-9]. For this type of intervention via MRI, there is also a method analogous to CT in which the movement of the instruments is not monitored interactively but rather planned movements of the instruments are monitored in a targeted manner via a few individual slices [10 - 12]. This method can be used for interstitial interventions involving targets that are difficult to reach, but the effort to reach these is higher than when using direct control of instrument movements. The lack of radiation exposure is a decisive advantage compared to CT-guided puncture, particularly for younger patients. MRguided intervention must be compared to ultrasound regarding effort and benefits. Even the smallest lesions can be clearly identified due to the high soft-tissue contrast of MR imaging. For punctures of the breast it has long been established to use MR-guided biopsy in lesions that cannot be visualized with other modalities [12]. This advantage resulted in the extensive clinical application of this method. Multiplanar slice orientation also allows puncture of target regions in a subdiaphragmatic location that are difficult to access. This is a major advantage, but is also possible with CT or ultrasound in skilled hands.

The limited number of open MRI units and the insufficient support from the industry are current disadvantages. MR-compatible intervention instruments are not boradly available. The technical principles of MR-guided intervention are not self-explanatory and must be communicated accordingly. Therefore, the further development and broader application of the method require close cooperation with the industry and the further development of suitable MRI equipment. To achieve this, clear indications are needed 
as the breast biopsy example shows. Most MR-guided interventions are currently not performed on open scanners as described in the section "Navigation".

\section{Prostate biopsy}

The standard method for diagnosing prostate cancer is transrectal ultrasound-guided (TRUS) multi-core biopsy of the prostate. In this case the term "ultrasound-guided" relates to the goal of biopsying certain areas of the prostate as an organ. There is usually not a concrete target that is visible on ultrasound. In the case of repeatedly negative TRUS results, saturation biopsy, in which the organ is systematically punctured in all regions to detect prostate cancer, is being increasingly performed. The detection rate for prostate cancer for TRUS is between $22 \%$ and $56 \%$ depending on patient selection with up to $40 \%$ of cancers diagnosed in this way having a Gleason score of $\leq 6$, i. e., being less biologically/prognostically relevant according to current interpretation. Prostate cancer in the anterior stroma, the anterior transition zone, and the lateral peripheral zone, as well as apical and basal prostate cancer cannot be diagnosed with TRUS [13 - 15]. In contrast, multiparametric MRI of the prostate provides a number of decisive advantages: It allows the detection of biologically significant prostate cancer (Gleason >6) with significantly higher reliability than TRUS with published sensitivity rates of up to $94 \%$. With increased understanding of the overdiagnosis and overtreatment of prostate cancers with minimal biological relevance, a further significant advantage of prostate MRI is that it has significantly lower sensitivity for biologically (prognostically) irrelevant cancers compared to the conventional approach. Finally, it should at least be mentioned that MRI is noninvasive [16-22].

The use of MRI to diagnose increasing PSA values after a negative TRUS result has since been included in the guidelines. Due to the above described sensitivity for biologically relevant prostate cancers, the inclusion of MRI in the guidelines for active surveillance is currently being discussed. Following some recently published larger studies, a fundamental shift in paradigm regarding the diagnosis of prostate cancer can be expected and MRI will be used instead of TRUS for primary diagnosis in patients with a rising PSA to increase the diagnosis of biologically relevant prostate cancers as well as to reduce the number of biologically irrelevant prostate cancers detected per blind biopsy [23].

If suspicious lesions are to be detected on mpMRI (multiparametric MRI), histological confirmation is necessary for further treatment planning. This confirmation can and should be performed via MR-guided prostate biopsy. Due to the low availability and high costs, "MRI/US fusion biopsy" is promoted instead of direct MR-guided prostate biopsy. In this method, the image information provided by MRI is electronically superimposed on a corresponding ultrasound image so that the biopsy can be performed with ultrasound guidance. Corresponding systems are becoming increasingly available. Urology specialists have also developed a method called "cognitive fusion" in which MRI findings are simply committed to memory for subsequent biopsy under ultrasound guidance. According to the literature, this approach does yield higher accuracy than conventional TRUS. The extent to which the accuracy of direct MR-guided biopsy is actually reached currently cannot be determined because of the lack of comparison studies.

In MR-guided biopsy of the prostate, a previously defined individual target lesion is biopsied in a targeted manner. Therefore, the procedure is significantly less traumatic compared to conventional blind biopsy. Transrectal or transperineal MR-guided biopsy can be performed. In MR-guided prostate biopsy, detection rates (PPV: positive predictive value) of $70 \%$ were published [22]. This corresponds with our own unpublished study with a detection rate of $71 \%$ in 134 transrectal MR-guided biopsies.

With appropriate preparation and interventional experience, MR-guided core biopsies of the prostate can be performed in 30 - 40 minutes. Depending on the access, patients lie in a prone or lithotomy position in the magnets. Prophylactic antibiotic therapy and intestinal voiding on the day of the intervention are recommended as in multi-core biopsy. Planning is usually based on T2-weighted sequences on at least two planes or in $3 \mathrm{D}$. Biopsy coordinates can be determined interactively or using planning software. In the case of a transrectal approach, 2 - 4 samples are extracted via an $18 \mathrm{G}$ core biopsy needle following insertion of a guide along the path of puncture. Multiple suspicious areas can be biopsied in one session. In contrast to ultrasound-guided "fusion biopsy", it is possible to visualize the needle position in relation to the location of the prostate cancer and to verify correct positioning in the lesion ( $\triangleright$ Fig. 2 ). The targeted approach in MRguided biopsy considerably reduces the number of biopsies needed to diagnose prostate cancer. Side effects (particularly prostatitis) are significantly rarer. In addition, all locations in the prostate are accessible. Oncologically speaking, the approach is significantly more productive since it ensures the diagnosis of biologically relevant prostate cancer requiring treatment and avoids the diagnosis of biologically irrelevant prostate cancer not requiring treatment [23, 24].

The combination of multiparametric MRI and MR-guided biopsy of the prostate currently represents the gold standard in the detection and confirmation of prostate cancer. In comparison to the diagnostic standard, i. e., multi-core biopsy, mpMRI has a number of advantages. Only findings that are visible on MRI should be biopsied under MR guidance. This should be viewed as the standard. Other methods should only be used if the accuracy of the biopsy will not be negatively affected. Given the acceptable intervention times in MR-guided prostate biopsy, the extent to which such "indirect MR-guided" biopsies are actually more costeffective is unclear.

\section{MR-guided breast interventions}

Since their introduction in the clinical routine at the end of the 1990 s, MR-guided breast interventions have been increasingly performed in Western industrialized nations as part of treatment $[25,26]$. This was facilitated by equipment development and is also the result of the broad use of MR mammography as a sensitive imaging method in the detection of breast cancer. When using modern high-end equipment, the sensitivity of MR mammography for detecting malignancy is $71-100 \%$ and the specifi- 

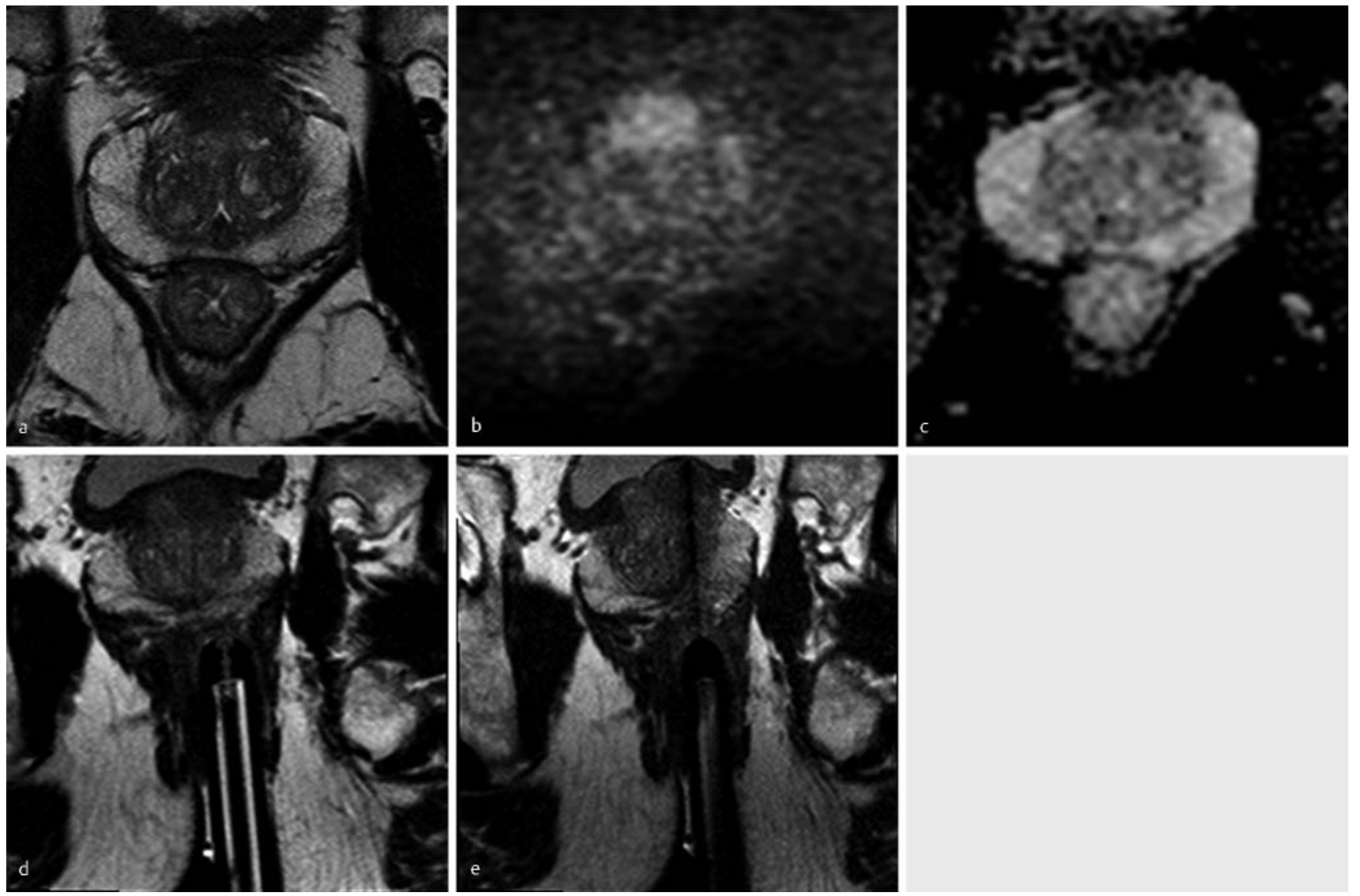

- Fig. 2 MR-guided Biopsie in a 66-years old patient with increasing PSA (last $6.5 \mathrm{ng} / \mathrm{ml}$ ) following multiple negative TRUS. A-C Diagnostic multi parametric MRI; Axial T2-TSE A, DWI $(b=1400 \mathrm{~mm} 2 / \mathrm{sec})$ B and ADC-MAP C demonstrate a highly suspicious region in the anterior stroma. MRguided biopsy of this area D, E. Histology: prostate cancer, Gleason $4+4=8$.

city is $81-99 \%$ [26]. The fact that an increasing number of BI-RADS 4/5 findings in MR mammography do not have a radiomorphological or sonographic correlate requires a histological workup via percutaneous biopsy or excisional biopsy [27, 28]. In principle, two different types of MR-guided breast intervention are used: preoperative marking of a lesion and percutaneous, usually vacuum-assisted biopsy.

The indication for performing MR-guided breast intervention should be determined on an interdisciplinary basis in a senology conference. MR-guided breast biopsy is indicated in BI-RADS 4/5 findings suspicious for malignancy without a sonographic or radio-mammographic correlate [27, 28,39$]$. Common indications are the preoperative workup of ipsilateral or contralateral MR-mammographic findings in cases of cancer already confirmed by histology, diagnostic confirmation of lesions suspicious for malignancy as part of the early detection program for hereditary breast cancer, expanded preventive care and follow-up of incidental breast cancer and the differentiation between post-therapeutic scars and tumor recurrence.

MR-guided wire marking is typically indicated in the case of lesions on MR-mammography that are not accessible for MR-guided biopsy due to their position near the nipple, skin or thoracic wall so that excisional biopsy must be performed.
The average intervention time is 30 to 40 minutes. The complication rate is up to $7 \%$ in vacuum-assisted biopsy and up to $5 \%$ in MR-guided wire marking with hematomas not requiring treatment, bleeding from the puncture channel, and pain at the puncture site being the most common complications [29, 30].

Finally the correlation between the histological results and the MR-mammographic findings must be determined in an interdisciplinary senology conference.

MR-guided interventions should be performed on high-field MRI systems at $1.5 \mathrm{~T}$ or $3 \mathrm{~T}$. The biopsy unit includes a dedicated breast coil, a compression system, and a localization system.

Dedicated MR-compatible biopsy systems from different manufacturers are available for MR-guided vacuum-assisted biopsy. The materials needed for MR-guided wire marking and clip marking are also available from various manufacturers.

The success rates are $96-100 \%$ for MR-guided vacuum-assisted biopsy and 91 - $100 \%$ for MR-guided wire marking [30 49]. The average rate of false-negative biopsies is $2 \%$ (between $0 \%$ and $13 \%$ ) [36-38].

In summary, MR-guided breast interventions have been firmly established in the senological routine for approximately a decade and will be applied more broadly in the future following an increase in the use of MR mammography. MR-guided breast inter- 
ventions are medically indicated in all BI-RADS 4/5 findings on MR mammography that are relevant for treatment decisions and have no sonographic or radio-mammographic correlate. Due to the significant requirements regarding equipment and experience, MR-guided breast interventions should be performed at designated centers.

\section{Instruments and new operating concepts for vascular interventions}

The possibility of performing vascular interventions under MR guidance is one of the oldest promises in MR imaging. After the initial introduction of promising approaches for interventions on the kidney [50], liver [51], and heart [52, 53], it took almost 8 years for a new generation of materials and MRI techniques to bring new possibilities. MR-guided vascular intervention is dependent on the availability of suitable catheters and guide wires. A further important component in the success of MR-guided vascular intervention is the MRI user interface for interventionalists.

At least two manufacturers are currently in the end phase of CE certification for MR-compatible guide wires at $1.5 \mathrm{~T}$ and $3 \mathrm{~T}$. Other manufacturers are working on approval for diagnostic catheters primarily for $1.5 \mathrm{~T}$ environments. The issues regarding electrical and mechanical safety in the case of MRI and possible solutions are understood by the small companies presently in the market. The current development phase of this new field is characterized by fundamentally different approaches regarding materials selection. The selected materials result in different levels of guide wire stiffness and are currently all still inferior to the materials typically used in DSA (digital subtraction angiography). However, the materials selection indicates dynamic development promising rapid progress. Larger arterial and venous vessels can be safely and gently probed with the currently available wires [54].

There are a number of approaches to the visualization of catheters and guides. The spectrum ranges from active tracking with individual active transmitter coils integrated in the catheter and discrete iron markers for passive visualization to wires and catheters doped over the entire length with iron particles.

Active tracking requires a small coil to be actively supplied with voltage over the length or at the tip of the catheter. The active coil generates a signal that can be locally resolved by the MRI unit. The information can be used to visualize the coil on an image or to center the scan plane at the position of the active catheter coil. The coil as well as the leads require space and change the mechanical properties of the catheter. Active tracking requires special software for the scanner. The coils make the catheter very expensive. Moreover, safety aspects must be taken into consideration. Continuous conductive connections cannot be used since they can result in uncontrollable heating with the risk of burns depending on their alignment with respect to the magnetic fields. There are various solutions to this problem such as the use of short segments connected by suitable elements (capacitors).

Passive tracking uses susceptibility artifacts caused by iron and other ferromagnetic and paramagnetic materials to mark the position of guide wires or catheters. The size and shape of susceptibility artifacts depend on the distribution and size of the par- ticles used. The type and parameters of the imaging sequences also affect the size and shape of the generated artifacts. Passive tracking is simple from a technical standpoint. The major advantage is, for example, the ability to visualize the entire course of a guide wire due to materials introduced over a long stretch, thus providing an overview of the catheter-wire combination in MRI as in DSA.

The debate regarding the advantages of active vs. passive tracking currently being conducted in publications will eventually be settled. It will become increasingly clear that both tracking methods are needed for the clinical routine [55]. Passive techniques tend to be used for guide wires and very fine catheters while a combination of active and passive techniques are used for diagnostic and treatment catheters.

Active tracking requires cooperation between catheter manufacturers and MRI suppliers, which significantly limits the speed of development in this area. With respect to MRI, European manufacturers have become the leaders in innovation.

The user interface for interactive work on the scanner is still in need of significant development. The currently available solutions are based on the conventional operating philosophies of MRI and CT units. There is a lack of control via characteristic buttons, levers, and dedicated navigation knobs. Therefore, the interventionalist must rely on a second person to navigate the imaging plane. This slows the process and critical maneuvers are avoided for safety reasons since fast reactions are not possible. The limited spatial and temporal resolution of available real-time imaging contributes to this. However, there are positive developments: The development teams of MRI manufacturers are working on the concept of a control panel on the MRI examination table with controls borrowed from DSA. The fact that MRI cannot be costeffectively used exclusively for interventions has also been recognized. Therefore, there is increased willingness to design the additional controls for interventions so that they can be easily removed from the MRI unit for diagnostic operation. New approaches in the sequencing technique allow high spatial and temporal resolutions [56] that can be combined with the total range of tissue contrasts in some cases [57].

This is the closest we've ever been to being able to use MRguided vascular intervention in the clinical routine.

\section{Endovascular and cardiac interventions}

MR-guided endovascular and cardiac interventions were identified as a worthwhile goal soon after the introduction of MRI in the clinical routine due to the good soft-tissue contrast, the lack of radiation exposure, the ability to directly visualize target regions, and the functional parameters measurable with MRI such as blood flow and cardiac function. The development effort needed to manufacture MR-safe wires and catheters for MR-guided endovascular interventions and to adapt the operation of the unit accordingly is quite significant. The introduction of the method at a hospital is also associated with high costs. Moreover, angiography-guided endovascular interventions are well established and are comparatively cost-efficient. These impediments can only be overcome in the case of interventions that are not possible 

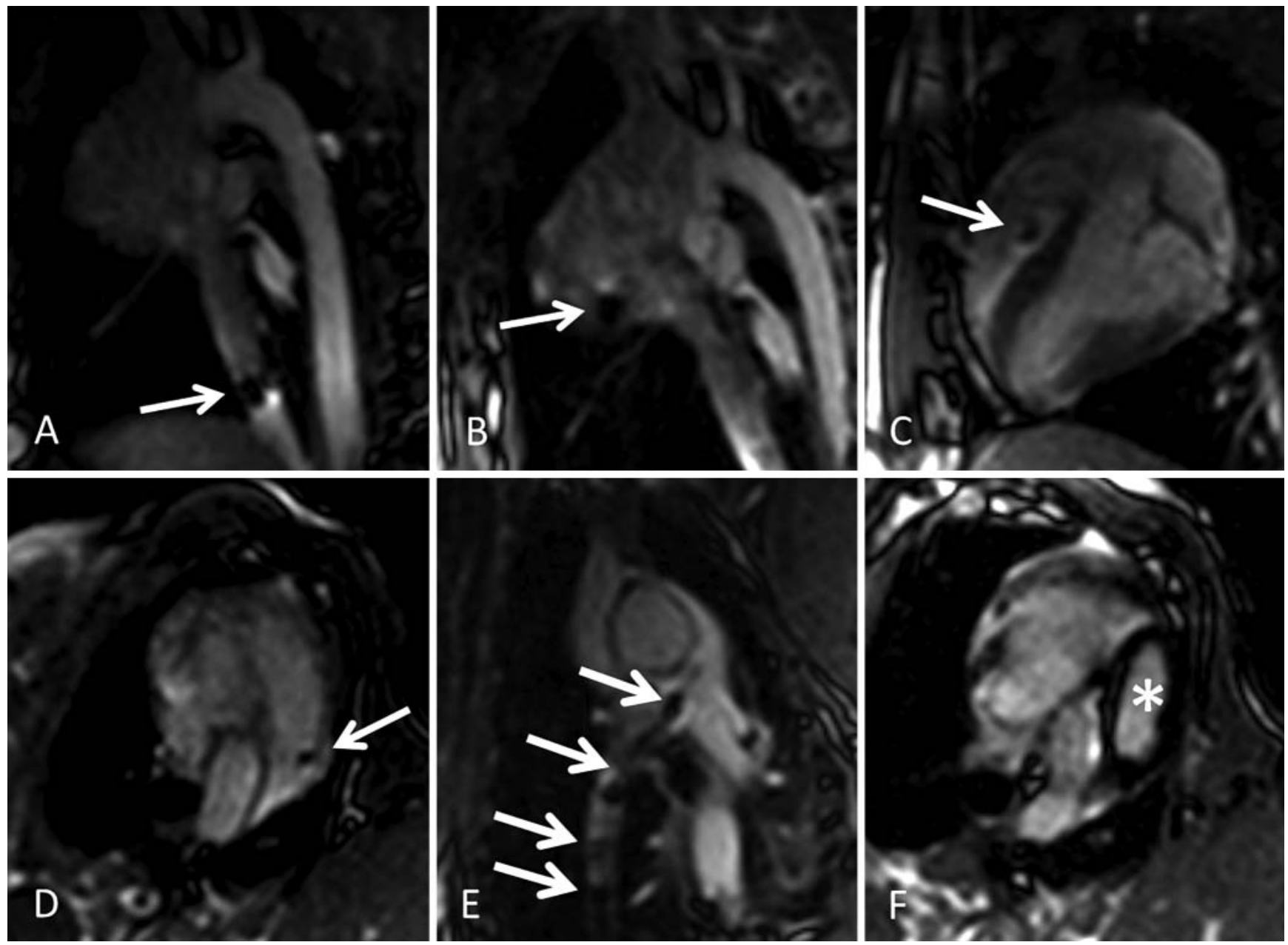

- Fig. 3 Dilatation of the pulmonary valve. A Swan Ganz ballon catheter, filled with $\mathrm{CO}_{2}$ (arrows $\mathbf{A}-\mathbf{D}$ ) is advanced through the IVC $\mathbf{A}$ into the right atrium $\mathbf{B}$ and the right ventricle $\mathbf{C}$ into the main pulmonary trunc $\mathbf{D}$. An MR safe guide-wire ist advanced through this catheter $\mathbf{E}$ and the catheter replaced with the ballon catheter for dilatation. This catheter is placed in the valve and inflated (asterisk) using contrast medium $\mathbf{F}$.

via angiography or for which MRI would have such significant advantages that the high expenditure would be justified. Interventions in children with congenital heart defects were initially included in this category [58-60] since these young patients require repeated interventions while they are growing so that it is important to use an imaging method without radiation exposure. Functional parameters such as ventricular ejection fraction and flow rates that can be measured by MRI can be taken into consideration for treatment decisions. Today, children with congenital heart defects typically enjoy a long life due to an optimized therapeutic strategy. While the child is growing, repeated, albeit simple, interventions are necessary in most cases ( $\vee$ Fig. $\mathbf{3}$ ). This increases the necessity to reduce the radiation exposure due to diagnosis and interventions to a minimum. However, after initial studies first on animal models and later on patients that were able to show feasibility and advantages [1, 61, 62], the use of endovascular MRI for this indication has barely been pursued in recent years due to a lack of availability of instruments and the effort and cost of adapting currently available units to endovascular interventions. To still be able to profit from the advantages of MRI,
MRI datasets are increasingly being superimposed on angiography images used for interventions [63, 64].

Further interesting application areas for endovascular interventions via interventional MRI include the local application of substances or cells such as chemoembolization or intramyocardial injection with the goal of tissue regeneration [65]. This is conditional upon the ability to directly visualize the target area. Feasibility studies in animal models have been performed for these indications ( $\triangleright$ Fig. 4). However, a transfer to the clinical routine has not occurred again due to the lack of availability of suitable interventional catheters and guide wires.

The use of hybrid systems in the form of MRI scanners that are connected to angiography units so that the patient is examined on the same examination table for both units allows the use of MRI datasets acquired immediately prior to the intervention for planning and performing angiography-guided interventions as well as the continuation of MR-guided interventions under fluoroscopy guidance as a backup method if necessary [1, 58, 59]. Such systems allow interventional MRI to be further included in the clinical routine. In this context it is particularly advantageous that 

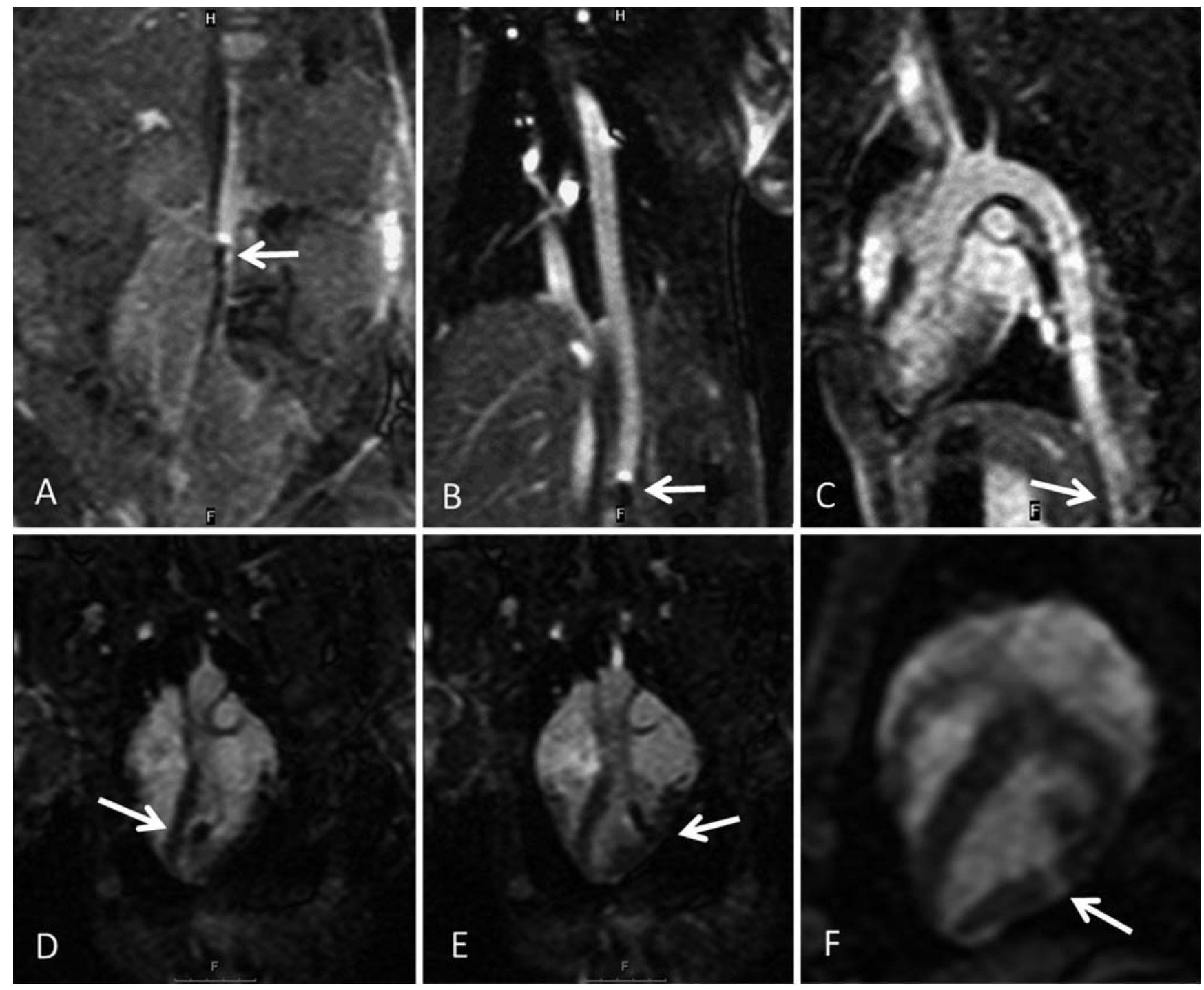

- Fig. 4 Intramyocardial injection for application of substances or cells. A The catheter (arrow) has been introduced via a sheath in the groin and advanced throught the abdominal aorta $\mathbf{A}$ into the thoracic aorta $\mathbf{B}$. For further advancing the catheter throught the aortic arch, slice position is adapted to the aortic arch $\mathbf{C}$. The tip off the catheter must be guidable for reaching all positions within the left ventricle $\mathbf{D}$, $\mathbf{E}$. This can be achieved by different cables, comparable to the mechanism employed in endoscopes. If the injectate is mixed with contrast medium, successful injection into the myocardium is visible by increase of signal intensity in T1-weighted images (asterisk, $\mathbf{F}$ ).

the dyna-CT function of angiography systems can be used to update the co-registration with an MRI dataset. As a result, MR guidance can be "exported" from the MRI unit in that the MRI dataset is co-registered with a second imaging method and the MRI dataset is used for interventions performed with the second imaging modality. The use of a hybrid unit would make it possible to position the patient table in the MRI gantry again in order to check the intervention success and to continue or end the intervention depending on the result of this check.

\section{MR-guided cardiac ablation}

The number of cardiac catheter ablation procedures and the pathophysiological understanding of complex arrhythmias are increasing steadily [66]. Visualization of the underlying substrate is decisive for the treatment of ventricular tachycardia and atrial fibrillation. Due to the $2 \mathrm{D}$ nature of $\mathrm{X}$-ray fluoroscopy, various $3 \mathrm{D}$ mapping systems using previously acquired $3 \mathrm{D}$ datasets from CT or MRI have been developed. However, these systems still have problems primarily with regard to the registration and fusion of datasets and therefore continue to require additional fluoroscopy [67].

In comparison, real-time MR imaging offers promising possibilities with respect to electrophysiological examination and ablation because

1. no ionizing radiation is used,

2. the intervention can be guided with a range of imaging information e. g. regarding fibrosis/necrosis/scars with late enhancement, post-therapeutically with edema-sensitive se- 

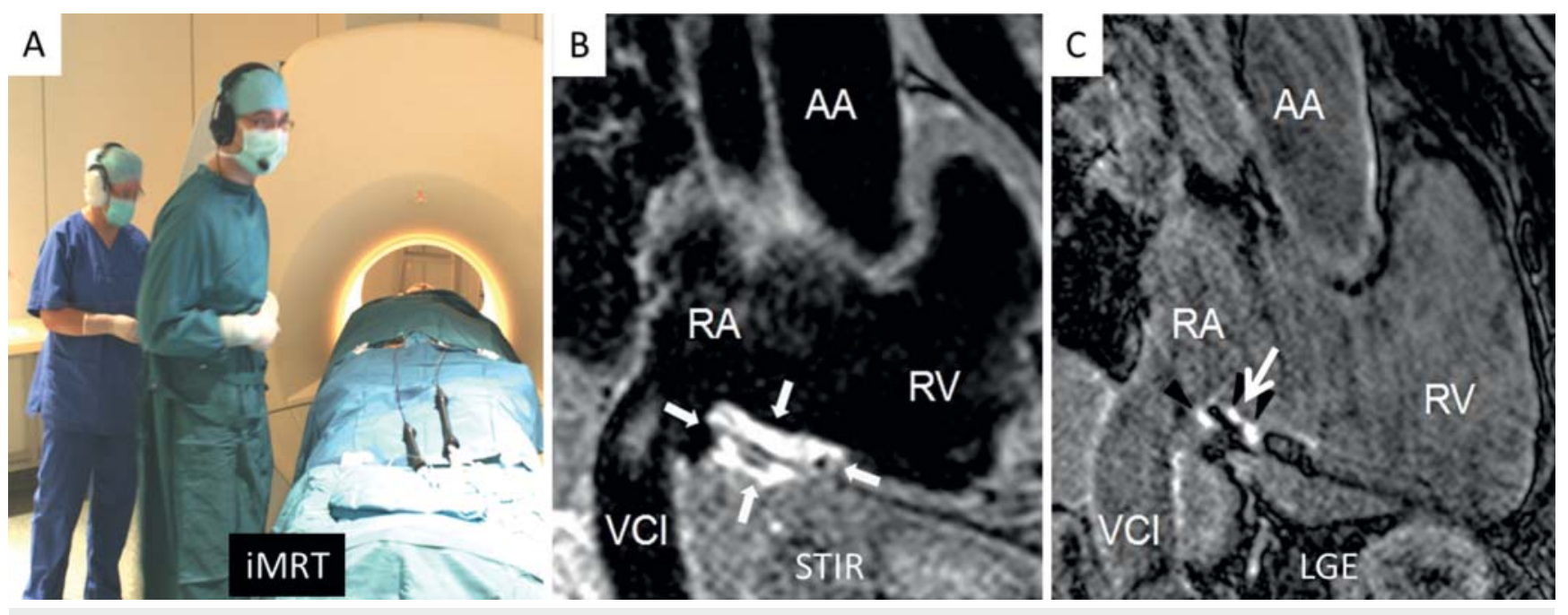

- Fig. 5 MR-guided Ablation of a focus for atrial fibrillation at the cavotricuspidal isthmus. A Intervention in a 1.5 T scanner with placed, MR conditional catheters (IMRICOR) and head sets for communication. Sucessful ablation with delineation of the edema in a STIR image (B, arrow) and the scar (C, arrow) on late enhancement IR_GRE and in RAO projection [67].

quences ( $\triangleright$ Fig. 5) and perspectively with thermosensitive sequences,

3. anatomical and functional information (in $3 \mathrm{D}$ or $4 \mathrm{D}$ ) can be acquired,

4. both the catheter (active or passive) and the ablated substrate can be visualized.

To date, MR-guided electrophysiological interventions have been largely limited to examinations and treatments in animal experiments. In recent years, however, initial simple electrophysiological studies [68] as well as ablation procedures [69, 70] have been performed, e. g. isthmus ablation of patients with atrial flutter under passive catheter guide [71] ( Fig. 5). Grothoff et al. [67] successfully performed this procedure in patients on a $1.5 \mathrm{~T} \mathrm{MRI}$ unit. A real-time SSFP sequence was used for imaging.

Especially in patients with ventricular tachycardia [72], MRI is the gold standard for substrate imaging and is accordingly being increasingly used. The presence of an implantable cardioverter defibrillator (ICD) is still a contraindication to MRI. However, in the future such patients could benefit from the development of MR-conditional implants under certain conditions [73].

Despite current limitations, MRI techniques will continue to develop both for risk stratification via preprocedural imaging and for MR-guided catheter ablation due to the clinical demand and the good results achieved to date. The next step in the treatment of simple arrhythmias, such as atrial flutter, is a combination of passive and active methods for catheter tracking. The position is determined almost in real time, allowing the catheter to be tracked within a previously acquired $3 \mathrm{D}$ dataset showing the entire heart in high resolution without having to perform imaging again.

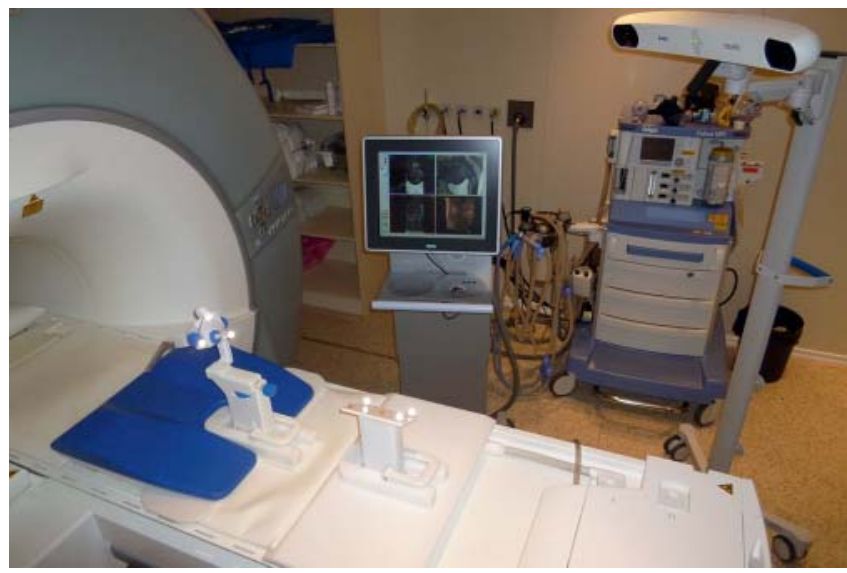

- Fig. 6 Navigation system for MR-guided biopsy of the prostate at a 3-T scanner. After registration of the biopsy device (Invivo, Schwerin, Germany) the navigation system (Localite, St. Augustin, Germany) reconstructs from the optically detected orientation of the biopsy device the corresponding MR slices and depicts them continuously on the in-room monitor.

\section{Navigation}

Guides and navigation aids find broad application in image-guided interventions. They range from simple components for holding and introducing needles and manipulators for setting a certain trajectory to full navigation systems with visual feedback and typically include special application software [74] ( Fig. 6). In contrast to ultrasound- and CT-guided methods, the strong basic magnetic field and the alternating electromagnetic fields place special requirements on components used in the MRI room. Among other things, this requires the use of special materials or does not allow the use of electromagnetic localization of the instrument. 
In particular, systems for breast [75] and prostate interventions [76] that allow precise puncture on the basis of MRI images acquired during the intervention are widely used. After suitable registration of the target unit, the needle trajectory is calculated directly from a graphically marked MRI position in some systems [77]. In addition, there are special solutions for other organ regions, such as the brain [78], the spine, or the musculoskeletal system [79]. In contrast, the navigation of instruments in moving organs, such as the liver, is significantly more challenging. This challenge is currently met by concepts for open MRI units allowing advance of the needle under real-time image guidance as described above [4].

In addition to a number of internal developments, there is currently increased commercial involvement including the production of specialized components and tools as well as the development of corresponding software applications. In addition, some equipment manufacturers offer special interfaces that allow the user to interactively control imaging for visualizing and adjusting the needle [80].

Although assistance systems are often not an indispensable part of MR-guided interventions, they are a useful addition. The main benefit is the improved workflow due to more targeted intervention planning, expanded visualization and additional control options. Beginners should profit from this in particular, and lower variability can be expected among experienced users.

The results regarding the accuracy and time expenditure of the method are fundamentally positive. However, irrefutable statements regarding improved clinical results are generally difficult to make. The relatively high development costs in connection with the relatively low number of installed systems and MR-guided interventions at specialized centers hinder large multi-center studies, required for such statements. Moreover, additional use is currently not compensated. However, due to the price trend of high-performance computers and display systems and the growing clinical experience with such systems, broader application can be anticipated in the medium term.

\section{Intraoperative use of interventional MRI}

Neuronavigation on the basis of MRI datasets has long been a standard technique for complete neurosurgical resection of intracerebral tumors [81]. "Brain shift", i. e., the change in the position of the brain after trepanation, the subsequent loss of CSF and the deformation of the tissue due to the resection of tumor segments, decreases the accuracy of neuronavigation. Intraoperative MRI can be used to generate current data for navigation [82] so that the tumor tissue can be removed as completely as possible and eloquent areas of the brain can be protected to the greatest extent possible [83]. In connection with neurosurgical instruments or with the fixed endoscope that is used in neurosurgery in some cases, the benefit can be further increased [84]. In neurosurgical interventions, interventional MRI facilitates the locating of target regions and the removal of tumors that is as complete as possible with the fewest possible side effects.

\section{Conclusion and outlook}

Interventional MRI is already established in the clinical routine for interstitial applications. Technical solutions have been created for almost every requirement but are often not commercially available despite their development maturity. A commercially available range of instruments, software and interfaces is the next necessary step in the introduction of these methods into the clinical routine. Hybrid systems in the form of a combination of MRI units and angiography units (with the option for rotational CT) can facilitate the incremental shift to MR-guided interventions.

\section{Conflict of Interest}

The authors declare that they have no conflict of interest.

\section{References}

[1] Tzifa A, Krombach GA, Kramer $\mathrm{N}$ et al. Magnetic resonance-guided cardiac interventions using magnetic resonance-compatible devices: a preclinical study and first-in-man congenital interventions. Circ Cardiovasc Interv 2010; 3: 585-592

[2] de Bucourt M, Streitparth F, Wonneberger $U$ et al. Obese patients in an open MRI at 1.0 Tesla: image quality, diagnostic impact and feasibility. Eur Radiol 2011; 21: 1004-1015

[3] Wonneberger U, Schnackenburg B, Streitparth F et al. Evaluation of magnetic resonance imaging-compatible needles and interactive sequences for musculoskeletal interventions using an open high-field magnetic resonance imaging scanner. Cardiovasc Intervent Radiol 2010; 33: $346-351$

[4] Fischbach F, Bunke J, Thormann M et al. MR-guided freehand biopsy of liver lesions with fast continuous imaging using a 1.0-T open MRI scanner: experience in 50 patients. Cardiovasc Intervent Radiol 2011; 34: $188-192$

[5] Fischbach F, Lohfink K, Gaffke $G$ et al. Magnetic resonance-guided freehand radiofrequency ablation of malignant liver lesions: a new simplified and time-efficient approach using an interactive open magnetic resonance scan platform and hepatocyte-specific contrast agent. Invest Radiol 2013; 48: 422-428

[6] Fischbach F, Porsch M, Krenzien F et al. MR imaging guided percutaneous nephrostomy using a 1.0 Tesla open MR scanner. Cardiovasc Intervent Radiol 2011; 34: 857-863

[7] Fischbach F, Eggemann H, Bunke J et al. MR-guided freehand biopsy of breast lesions in a 1.0-T open MR imager with a near-real-time interactive platform: preliminary experience. Radiology 2012; 265: 359-370

[8] Ricke ], Thormann M, Ludewig M et al. MR-guided liver tumor ablation employing open high-field 1.0T MRI for image-guided brachytherapy. Eur Radiol 2010; 20: 1985-1993

[9] Streitparth F, de Bucourt M, Hartwig T et al. Real-time MR-guided lumbosacral periradicular injection therapy using an open 1.0-T MRI system: an outcome study. Invest Radiol 2013; 48: 471-476

[10] Vogl T], Dommermuth A, Heinle B et al. Colorectal cancer liver metastases: long-term survival and progression-free survival after thermal ablation using magnetic resonance-guided laser-induced interstitial thermotherapy in 594 patients: analysis of prognostic factors. Invest Radiol 2014; 49: 48 - 56

[11] Salomonowitz E. MR imaging-guided biopsy and therapeutic intervention in a closed-configuration magnet: single-center series of 361 punctures. Am J Roentgenol 2001; 177: 159-163 
[12] Kuhl CK, Morakkabati N, Leutner CC et al. MR imaging-guided largecore (14-gauge) needle biopsy of small lesions visible at breast MR imaging alone. Radiology 2001; 220: 31 - 39

[13] Franiel T. Multiparametric magnetic resonance imaging of the prostate - technique and clinical applications. Fortschr Röntgenstr 2011; 183: $607-617$

[14] Turkbey B, Choyke PL. Multiparametric MRI and prostate cancer diagnosis and risk stratification. Curr Opin Urol 2012; 22: 310-315

[15] Fradet V, Kurhanewicz J, Cowan JE et al. Prostate cancer managed with active surveillance: role of anatomic MR imaging and MR spectroscopic imaging. Radiology 2010; 256: $176-183$

[16] Siddiqui MM, Rais-Bahrami S, Turkbey B et al. Comparison of MR/ultrasound fusion-guided biopsy with ultrasound-guided biopsy for the diagnosis of prostate cancer. JAMA 2015; 313: 390 - 397

[17] Hoeks CM, Vos EK, Bomers JG et al. Diffusion-weighted magnetic resonance imaging in the prostate transition zone: histopathological validation using magnetic resonance-guided biopsy specimens. Invest Radiol 2013; 48: $693-701$

[18] Hoeks CM, Barentsz JO, Hambrock T et al. multiparametric MR imaging for detection, localization, and staging. Radiology 2011; 261: 46-66

[19] Cornud F, Delongchamps NB, Mozer P et al. Value of multiparametric MRI in the work-up of prostate cancer. Curr Urol Rep 2012; 13: 82 - 92

[20] Schlemmer HP. Multiparametric MRI of the prostate: method for early detection of prostate cancer? Fortschr Röntgenstr 2010; 182: 1067 1075

[21] Djavan B, Milani S, Remzi M. Prostate biopsy: who, how and when. An update. Can J Urol 2005; 12: 44-48

[22] Eichler K, Hempel S, Wilby J et al. Diagnostic value of systematic biopsy methods in the investigation of prostate cancer: a systematic review. J Urol 2006; 175: 1605-1612

[23] Pokorny MR, de RM, Duncan E et al. Prospective study of diagnostic accuracy comparing prostate cancer detection by transrectal ultrasoundguided biopsy versus magnetic resonance (MR) imaging with subsequent MR-guided biopsy in men without previous prostate biopsies. Eur Urol 2014; 66: $22-29$

[24] Wagenlehner FM, van OE, Tenke P et al. Infective complications after prostate biopsy: outcome of the Global Prevalence Study of Infections in Urology (GPIU) 2010 and 2011, a prospective multinational multicentre prostate biopsy study. Eur Urol 2013; 63: 521 - 527

[25] Kuhl CK. Current status of breast MR imaging. Part 2. Clinical applications. Radiology 2007; 244: 672-691

[26] Mann RM, Kuhl CK, Kinkel K et al. Breast MRI: guidelines from the European Society of Breast Imaging. Eur Radiol 2008; 18: 1307 - 1318

[27] American College of Radiology (ACR). ACR practice guideline for the performance of magnetic resonance imaging-guided breast interventional procedures. http://www.acr.org/ /media/ACR/Documents/ PGTS/guidelines/MRI_Guided_Breast.pdf (letzter Zugriff 14 April 2016)

[28] AWMF, Deutsche Krebsgesellschaft e. V., Deutsche Krebshilfe e.V. Interdisziplinäre S3-Leitlinie für die Diagnostik, Therapie und Nachsorge des Mammakarzinoms. Aktualisierung 2012. http://www.krebsgesellschaft. de/download/S3_Brustkrebs_Update_2012_OL_Langversion.pdf (letzter Zugriff 14 April 2016)

[29] Liberman L, Bracero N, Morris E et al. MRI-guided 9-gauge vacuum-assisted breast biopsy: initial clinical experience. Am J Roentgenol 2005; 185: $183-193$

[30] Orel SG, Rosen M, Mies C et al. MR imaging-guided 9-gauge vacuumassisted core-needle breast biopsy: initial experience. Radiology 2006; 238: $54-61$

[31] Meeuwis C, Veltman J, van Hall HN et al. MR-guided breast biopsy at 3T: diagnostic yield of large core needle biopsy compared with vacuum-assisted biopsy. Eur Radiol 2012; 22: 341 - 349
[32] Noroozian M, Gombos EC, Chikarmane S et al. Factors that impact the duration of MRI-guided core needle biopsy. Am J Roentgenol 2010; 194: W150-W157

[33] Perlet C, Heywang-Kobrunner SH, Heinig A et al. Magnetic resonanceguided, vacuum-assisted breast biopsy: results from a European multicenter study of 538 lesions. Cancer 2006; 106: 982 - 990

[34] Friedman P, Enis S, Pinyard J. Magnetic resonance imaging-guided vacuum-assisted breast biopsy: an initial experience in a community hospital. Can Assoc Radiol J 2009; 60: $196-200$

[35] Fischer U, Kopka L, Grabbe E. Magnetic resonance guided localization and biopsy of suspicious breast lesions. Top Magn Reson Imaging 1998; 9: $44-59$

[36] Schrading S, Simon B, Braun M et al. MRI-guided breast biopsy: influence of choice of vacuum biopsy system on the mode of biopsy of MRI-only suspicious breast lesions. Am J Roentgenol 2010; 194: 1650-1657

[37] Rauch GM, Dogan BE, Smith TB et al. Outcome analysis of 9-gauge MRIguided vacuum-assisted core needle breast biopsies. Am J Roentgenol 2012; 198: $292-299$

[38] Shaylor SD, Heller SL, Melsaether AN et al. Short interval follow-up after a benign concordant MR-guided vacuum assisted breast biopsy - is it worthwhile? Eur Radiol 2014; 24: 1176 - 1185

[39] Heywang-Kobrunner SH. Contrast-enhanced magnetic resonance imaging of the breast. Invest Radiol 1994; 29: $94-104$

[40] Orel SG, Schnall MD, Newman RW et al. MR imaging-guided localization and biopsy of breast lesions: initial experience. Radiology 1994; 193: $97-102$

[41] Kuhl CK, Elevelt A, Leutner CC et al. Interventional breast MR imaging: clinical use of a stereotactic localization and biopsy device. Radiology 1997; 204: $667-675$

[42] Daniel BL, Birdwell RL, Ikeda DM et al. Breast lesion localization: a freehand, interactive MR imaging-guided technique. Radiology 1998; 207 : $455-463$

[43] Bedrosian I, Schlencker ], Spitz FR et al. Magnetic resonance imagingguided biopsy of mammographically and clinically occult breast lesions. Ann Surg Oncol 2002; 9: 457-461

[44] Morris EA, Liberman L, Dershaw DD et al. Preoperative MR imagingguided needle localization of breast lesions. Am J Roentgenol 2002; 178 : $1211-1220$

[45] Lampe D, Hefler L, Alberich T et al. The clinical value of preoperative wire localization of breast lesions by magnetic resonance imaging-a multicenter study. Breast Cancer Res Treat 2002; 75: 175-179

[46] Friedman P, Sanders L, Russo J et al. Detection and localization of occult lesions using breast magnetic resonance imaging: initial experience in a community hospital. Acad Radiol 2005; 12: $728-738$

[47] Landheer ML, Veltman J, van ER et al. MRI-guided preoperative wire localization of nonpalpable breast lesions. Clin Imaging 2006; 30: 229 233

[48] Gossmann A, Bangard C, Warm M et al. Real-time MR-guided wire localization of breast lesions by using an open 1.0-T imager: initial experience. Radiology 2008; 247: 535-542

[49] Krug KB, Ulhaas A, Hellmich M et al. Impact of clinical and lesion characteristics on the results of MR-guided wire localizations of the breast using an open 1.0-T MRI system. Invest Radiol 2013; 48: 445-451

[50] Fink C, Bock M, Umathum R et al. Renal embolization: feasibility of magnetic resonance-guidance using active catheter tracking and intraarterial magnetic resonance angiography. Invest Radiol 2004; 39: $111-119$

[51] Seppenwoolde JH, Bartels LW, van der Weide R et al. Fully MR-guided hepatic artery catheterization for selective drug delivery: a feasibility study in pigs. J Magn Reson Imaging 2006; 23: 123-129 
[52] Spuentrup E, Ruebben A, Schaeffter T et al. Magnetic resonance - guided coronary artery stent placement in a swine model. Circulation 2002; 105: 874-879

[53] Qiu B, Gao F, Karmarkar P et al. Intracoronary MR imaging using a 0.014inch MR imaging-guidewire: toward MRI-guided coronary interventions. J Magn Reson Imaging 2008; 28: 515-518

[54] Neizel M, Kramer N, Schutte A et al. Magnetic resonance imaging of the cardiac venous system and magnetic resonance-guided intubation of the coronary sinus in swine: a feasibility study. Invest Radiol 2010; 45: $502-506$

[55] Hilbert S, Sommer P, Gutberlet M et al. Real-time magnetic resonanceguided ablation of typical right atrial flutter using a combination of active catheter tracking and passive catheter visualization in man: initial results from a consecutive patient series. Europace 2016; 18: 572-577

[56] Voit D, Zhang S, Unterberg-Buchwald C et al. Real-time cardiovascular magnetic resonance at $1.5 \mathrm{~T}$ using balanced SSFP and $40 \mathrm{~ms}$ resolution. J Cardiovasc Magn Reson 2013; 15: 79

[57] Joseph A, Kowallick JT, Merboldt KD et al. Real-time flow MRI of the aorta at a resolution of 40 msec. J Magn Reson Imaging 2014; 40: 206-213

[58] Tzifa A, Schaeffter T, Razavi R. MR imaging-guided cardiovascular interventions in young children. Magn Reson Imaging Clin N Am 2012; 20: $117-128$

[59] Valverde I, Hussain T, Razavi R. Novel imaging techniques for the diagnosis and treatment of congenital heart defects: MR-guided interventions and beyond. Future Cardiol 2012; 8: 149-152

[60] Krueger J], Ewert P, Yilmaz $S$ et al. Magnetic resonance imaging-guided balloon angioplasty of coarctation of the aorta: a pilot study. Circulation 2006; 113: 1093 - 1100

[61] Moore P. MRI-guided congenital cardiac catheterization and intervention: the future? Catheter Cardiovasc Interv 2005; 66: 1-8

[62] Razavi R, Hill DL, Keevil SF et al. Cardiac catheterisation guided by MRI in children and adults with congenital heart disease. Lancet 2003; 362: $1877-1882$

[63] Abu Hazeem AA, Dori Y, Whitehead KK et al. X-ray magnetic resonance fusion modality may reduce radiation exposure and contrast dose in diagnostic cardiac catheterization of congenital heart disease. Catheter Cardiovasc Interv 2014; 84: 795-800

[64] Downing TE, Dori Y, Harris MA et al. successful transcatheter device occlusion using magnetic resonance imaging $X$-ray fusion. Congenit Heart Dis 2014; 9: E199-E203

[65] Krombach GA, Pfeffer JG, Kinzel S et al. MR-guided percutaneous intramyocardial injection with an MR-compatible catheter: feasibility and changes in T1 values after injection of extracellular contrast medium in pigs. Radiology 2005; 235: 487-494

[66] Eitel C, Hindricks G, Grothoff M et al. Catheter ablation guided by realtime MRI. Curr Cardiol Rep 2014; 16: 511

[67] Grothoff M, Piorkowski C, Eitel C et al. MR imaging-guided electrophysiological ablation studies in humans with passive catheter tracking: initial results. Radiology 2014; 271: 695-702

[68] Eitel C, Piorkowski C, Hindricks $G$ et al. Electrophysiology study guided by real-time magnetic resonance imaging. Eur Heart J 2012; 33: 1975

[69] Nordbeck P, Hiller KH, Fidler F et al. Feasibility of contrast-enhanced and nonenhanced MRI for intraprocedural and postprocedural lesion visuali- zation in interventional electrophysiology: animal studies and early delineation of isthmus ablation lesions in patients with typical atrial flutter. Circ Cardiovasc Imaging 2011; 4: 282-294

[70] Piorkowski C, Grothoff M, Gaspar T et al. Cavotricuspid isthmus ablation guided by real-time magnetic resonance imaging. Circ Arrhythm Electrophysiol 2013; 6: e7-e10

[71] Sommer P, Grothoff M, Eitel C et al. Feasibility of real-time magnetic resonance imaging-guided electrophysiology studies in humans. Europace 2013; 15: $101-108$

[72] Nazarian S, Bluemke DA, Lardo AC et al. Magnetic resonance assessment of the substrate for inducible ventricular tachycardia in nonischemic cardiomyopathy. Circulation 2005; 112: $2821-2825$

[73] Stevens SM, Tung R, Rashid S et al. Device artifact reduction for magnetic resonance imaging of patients with implantable cardioverter-defibrillators and ventricular tachycardia: late gadolinium enhancement correlation with electroanatomic mapping. Heart Rhythm 2014; 11: 289-298

[74] Moche M, Trampel R, Kahn T et al. Navigation concepts for MR imageguided interventions. J Magn Reson Imaging 2008; 27: 276-291

[75] Meeuwis C, Mann RM, Mus RD et al. MRI-guided breast biopsy at 3T using a dedicated large core biopsy set: feasibility and initial results. Eur J Radiol 2011; 79: 257-261

[76] Tilak G, Tuncali K, Song SE et al. 3T MR-guided in-bore transperineal prostate biopsy: A comparison of robotic and manual needle-guidance templates. J Magn Reson Imaging 2015; 42: 63-71

[77] Busse H, Garnov N, Thormer $G$ et al. Flexible add-on solution for MR image-guided interventions in a closed-bore scanner environment. Magn Reson Med 2010; 64: 922 - 928

[78] Martin AJ, Hall WA, Roark C et al. Minimally invasive precision brain access using prospective stereotaxy and a trajectory guide. J Magn Reson Imaging 2008; 27: 737-743

[79] Busse H, Kahn T, Moche M. Navigation concepts for magnetic resonance imaging-guided musculoskeletal interventions. Top Magn Reson Imaging 2011; 22: 179- 188

[80] Rothgang E, Gilson WD, Wacker F et al. Rapid freehand MR-guided percutaneous needle interventions: an image-based approach to improve workflow and feasibility. J Magn Reson Imaging 2013; 37: 1202-1212

[81] Krombach GA, Spetzger $U$, Rohde $V$ et al. Intraoperative localization of functional regions in the sensorimotor cortex by neuronavigation and cortical mapping. Comput Aided Surg 1998; 3: 64-73

[82] Torcuator RG, Hulou MM, Chavakula V et al. Intraoperative real-time MRI-guided stereotactic biopsy followed by laser thermal ablation for progressive brain metastases after radiosurgery. J Clin Neurosci 2016; 24: $68-73$

[83] Roder C, Charyasz-Leks E, Breitkopf M et al. Resting-state functional MR in an intraoperative MRI setting: proof of feasibility and correlation to clinical outcome of patients. J Neurosurg 2016: 1 -9

[84] Sylvester PT, Evans JA, Zipfel G] et al. Combined high-field intraoperative magnetic resonance imaging and endoscopy increase extent of resection and progression-free survival for pituitary adenomas. Pituitary 2015; 18: $72-85$ 
